\title{
Modelling and feedback control of vortex shedding for drag reduction of a turbulent bluff body wake
}

\author{
R. D. Brackston ${ }^{\mathrm{a}, \mathrm{b}}$, A. Wynn ${ }^{\mathrm{a}}$, J. F. Morrison ${ }^{\mathrm{a}}$ \\ ${ }^{a}$ Dept. Aeronautics, Imperial College London, SW7 2AZ, United Kingdom \\ ${ }^{b}$ Dept. Life Sciences, Imperial College London, SW7 2AZ, United Kingdom
}

\begin{abstract}
Three-dimensional bluff body wakes are of key importance due to their relevance to the automotive industry. Such wakes have both large pressure drag and a number of coherent flow structures associated with them. Depending on the geometry, these structures may include both a bistability resulting from a spatial symmetry breaking (SB), and a quasi-periodic vortex shedding. The authors have recently shown that the bistability may be modelled by a Langevin equation and that this model enables the design of a feedback control strategy that efficiently reduces the drag through suppression of asymmetry. In this work the stochastic modelling approach is extended to the vortex shedding, capturing qualitatively both the forced and unforced behaviour. A control strategy is then presented that makes use of the frequency response of the wake, and aims to reduce the measured fluctuations associated with the vortex shedding. The strategy proves to be effective at suppressing fluctuations within specific frequency ranges but, due to amplification of disturbances at other frequencies, is unable to give drag reduction.
\end{abstract}

Keywords: flow control, drag reduction, wakes, vortex shedding

\section{Introduction}

The flow over three-dimensional bluff bodies is of particular interest due to its relevance to automotive vehicles. Such bodies experience large pressure drag due to the large region of separated flow in the wake. For automotive vehicles operating at motorway speeds, this pressure drag is responsible for a significant proportion of the fuel consumption, therefore its reduction is a topic of key interest.

Three-dimensional bluff body wakes exhibit a number of key coherent structures, two of which are the static asymmetry and the quasi-oscillatory vortex shedding. Both features arise initially at very low Reynolds numbers $(R e)$ as respectively a reflectional symmetry breaking (SB) mode (Grandemange et al., 2012), and a temporal symmetry breaking, yet both are also observed at the much higher Reynolds numbers typical of road vehicles. In the flows over rectilinear bluff bodies, the SB mode is observed as an instantaneous asymmetry, mainly appearing in the recirculation region as displayed in figure 1(a). Under aligned flow conditions the wake flips randomly between two asymmetric states, each mirror symmetric with respect to the other, a phenomenon known as wake bistability. The instantaneous asymmetry of the wake leads to a lateral force and contributes to the pressure drag on the body. The feature is quite general, having been observed for a range of threedimensional bluff bodies of widely varying geometries (Rigas et al., 2015). Superposed on this, the vortex shedding is seen as quasi-periodic oscillations of the wake, occurring in both the lateral and the vertical dimensions (Grandemange et al., 2013b;

Email address: r.brackston13@imperial.ac.uk (R. D. Brackston)
Volpe et al., 2015). The relative importance of these two features with respect to the pressure drag on the body remains unclear.

The objective of this research is to develop efficient drag reduction methods for three-dimensional bluff bodies, without making large geometric modifications. A promising method for doing this involves suppression of coherent structures, including the SB mode and vortex shedding. Some passive methods such as a control cylinder in the wake (Cadot et al., 2015), base cavity (Evrard et al., 2015) or perimetric slit (García de la Cruz et al., 2017) have already shown promising results for both the suppression of unsteadiness and pressure drag reduction. However while effective, such passive methods may involve large geometric modifications and the exact requirements for suppression of the coherent structures remains unclear. This motivates a careful analysis of the physics of coherent structures in the wake, and the use of active feedback control for their suppression. In this work we develop a stochastic model to describe the vortex shedding in the wake before making use of the model in feedback control design.

In the recent work of Brackston et al. (2016) a stochastic modelling approach was developed for the bistability arising from the SB mode of the flow. This model was then used to develop a feedback control strategy that ultimately proved energetically efficient at drag reduction. Similar drag reduction has also been achieved in other studies (Li et al., 2016) targeting the asymmetry of the wake. Such studies aimed to reduce the quasi-static asymmetry associated with the SB mode, but have the knock-on effect of increasing unsteadiness at some frequencies. In this work we target the unsteadiness of the vortex shedding directly, and further seek to quantify the effect of fluc- 
tuations on the drag.

The control of vortex shedding has been pursued before, although this has generally tended to be at low Reynolds number and for predominantly two-dimensional flows. For example, early experimental work was conducted for control of the laminar flow over a circular cylinder by Ffowcs Williams and Zhao (1989); Roussopoulos and Monkewitz (1996) and was found to be effective. Such studies tended to empirically obtain controllers that were effective at suppressing the shedding, a methodology continued in more recent numerical studies by Son et al. (e.g. 2011). Other numerical studies have applied a more model based approach in which low-order, linear models of the flow are obtained from numerical simulations (Illingworth et al., 2014; Illingworth, 2016; Flinois and Morgans, 2016). While the controllers resulting from these models prove effective, applying them experimentally and at high Reynolds numbers is impractical. The only high (turbulent) Re implementation that the authors are aware of is from Pastoor et al. (2008), who suppressed the shedding over a two-dimensional D-shaped body at $R e_{D} \approx O\left(10^{4}\right)$. In this work, we attempt the controlled suppression of vortex shedding for a flow that is both fully three-dimensional and at high Reynolds number $O\left(10^{5}\right)$.

The paper is structured as follows. In section 2 we describe the experiment consisting of an Ahmed body fitted with dynamic forcing flaps. We then go on to describe the stochastic modelling in section 3, before detailing the ensuing control design and analysis methodology in section 4. Conclusions are finally given in section 5 .

\section{Experimental setup}

Investigations and feedback control are implemented experimentally on a scaled down, flat-back Ahmed body (Ahmed et al., 1984) of the proportions used in many other studies (Grandemange et al., 2012; Cadot et al., 2015), giving $R e$ of $O\left(10^{5}\right)$. This experimental setup is shown in figure 1(b). A force balance measures the total force and moment acting on the body while 8 Endevco $8507 \mathrm{C}$ pressure transducers take fluctuating pressure measurements, and an ESP-DTC pressure scanner supplies 64 static pressure measurements distributed over the model base. Forcing of the wake is achieved using two flaps located at the trailing edges of the body, each $19 \mathrm{~mm}$ in streamwise length and running the length of the edges to which they are mounted. In this work, the flaps are positioned on the top and bottom edges for control of the vortex shedding in the vertical plane. The flaps are mounted on hinges and driven by internal motors that regulate their motion. The angle of each flap $(\theta)$ is measured using a 12 bit magnetic encoder while the power supplied to the flaps is monitored through measurement of the supply voltage and current. Further details of the experimental setup may be found in Brackston (2017).

All experiments reported here were performed in the Donald Campbell wind tunnel of the Department of Aeronautics, with a free-stream velocity $U_{\infty}$ set to $15 \mathrm{~ms}^{-1}$ and controlled with a PID algorithm to within $0.25 \%$. The static pressure tappings were sampled at $250 \mathrm{~Hz}$ while all measurements were sampled at $25 \mathrm{kHz}$.
In order to quantify our measurement of the vertical vortex shedding, we take an antisymmetric component of the pressure measurement over the base of the body. This pressure metric, here referred to as $m_{v}$, is defined such that it may be evaluated from either the 8 pressure transducers or the 64 tappings, depending on the context (real-time or post-processing). This is achieved by choosing a normalised spatial function on which the pressure measurements are projected. This function $\phi_{v}(z)$ is defined as,

$$
\phi_{v}(z)=\frac{\pi z}{H} /\left|\frac{\pi z}{H}\right|,
$$

where $|\cdot|$ denotes the 1 -norm of a vector. Given any set of $n$ pressure measurement $\boldsymbol{p}=\left[p_{1}, p_{2}, \ldots p_{n}\right]$, at vertical positions $z=\left[z_{1}, z_{2}, \ldots z_{n}\right]$, the vertical pressure mode is evaluated as the dot product,

$$
m_{v}(t)=\boldsymbol{p} \cdot \boldsymbol{\phi}_{v}
$$

\section{Stochastic modelling of coherent structures}

A first step in the understanding and control of coherent structures in a turbulent flow, is the development of low-order models for their dynamics. Such models may then give insight into the nature of feedback control strategies that may then be applied. A promising approach is based upon the observation that these coherent structures in the turbulent flow are often the persistence of the bifurcations seen at very low Re (Rigas et al., 2014). This allows the development of phenomenological models that make use of the equations governing these low $R e$ bifurcations, with the addition of stochastic terms to model the effect of turbulent fluctuations. We will begin by recapping the application of such an approach to the modelling of the SB mode (Brackston et al., 2016), before extending the method to the vortex shedding.

\subsection{Bistability}

For the Ahmed body of this experiment, the SB mode gives a bistable wake in the lateral dimension (Grandemange et al., 2013a). Applying the stochastic modelling approach, we may write an equation for the SB mode of the wake, quantified by a metric $r$, as the equation governing a pitchfork bifurcation with the addition of a stochastic forcing. Additionally the influence of forcing flaps is included via a term $b \theta_{t-\tau}$, capturing the linear influence of the flaps and incorporating an advective time delay $\tau$ :

$$
\dot{r}=\alpha r-\lambda r^{3}+b \theta_{t-\tau}+\sigma \xi(t)
$$

As detailed in Brackston et al. (2016), the parameters from this model may be found by examining both time-averaged and time-resolved data: the probability distribution (PDF) of the metric $r$ under a range of $\theta$ and the frequency response between $\theta$ and $r$. It is also important to note that the model accurately captures the forced response of the wake. Through static offsets the flaps can induce the wake to remain in one or other of the two asymmetric states, an effect that is captured by a FokkerPlanck equation with non-zero $\theta$. Under harmonic forcing the flaps induce repeated flipping of the wake between these two 


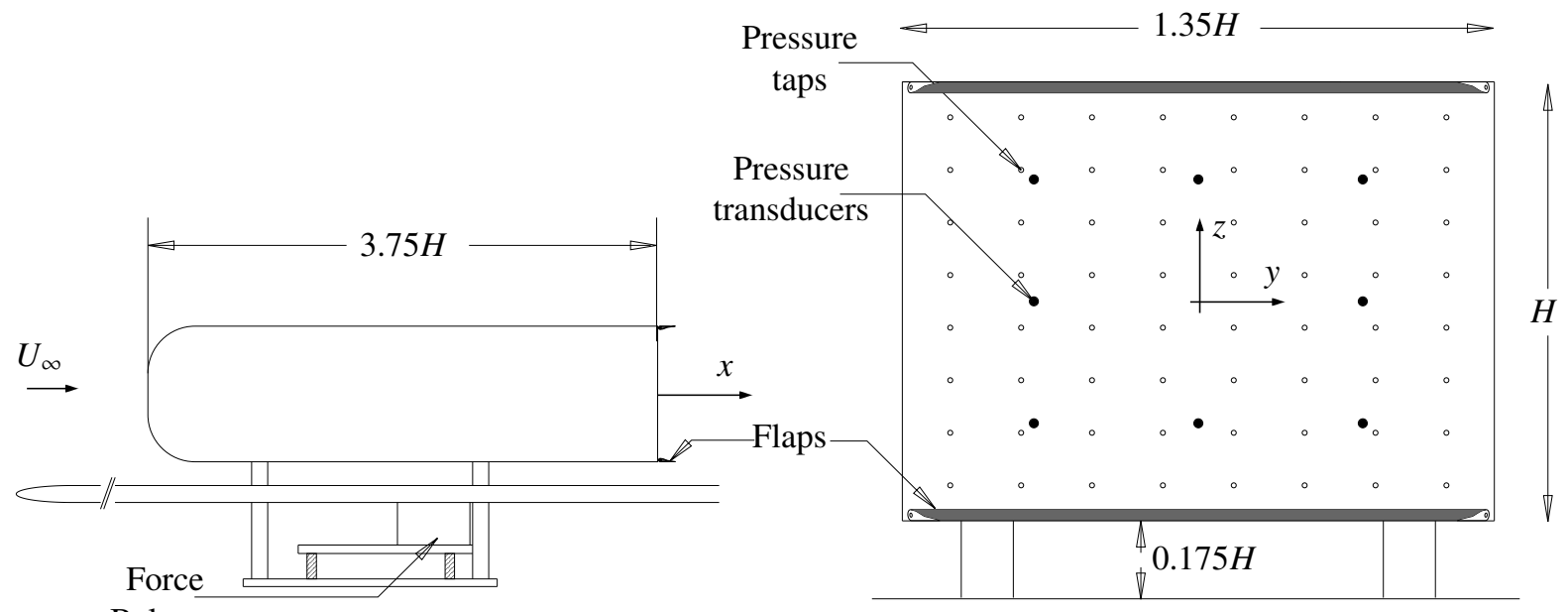

Balance

Figure 1: Side and rear view of the Ahmed body showing the locations of the flaps, pressure measurements and force balance. The flaps are to the top and bottom edges of the rear of the body and driven by internal motors. The model height $H$ is $0.16 \mathrm{~m}$.

states. Under such forcing the measured response acts something like a 1st-order low-pass filter, with a large response at low frequencies that reduces as the forcing frequency increases above $\alpha$. For feedback control purposes, sufficient accuracy with respect to the forced response is important for effective control design.

\subsection{Vortex Shedding}

The general stochastic modelling approach may also be extended to the vortex shedding in the wake. This vortex shedding manifests as quasi-oscillatory unsteadiness of the wake in both the top-down (vertical) and left-right (lateral) dimensions (Volpe et al., 2015), and is visible as broadband fluctuations in the base pressure spectrum. A model is therefore required that can display both broadband self-excited oscillations when unforced, as well as resonant behaviour under the influence of forcing (Barros et al., 2016; Rigas et al., 2017). Unlike for the bistability, we will not seek to find a model that accurately reproduces the behaviour seen experimentally in a quantitative sense, but rather demonstrate that the general form of model proposed here may qualitatively capture the key characteristics.

For the vortex shedding behind bluff bodies, the initial bifurcation is a Hopf bifurcation (Jackson, 1987), leading to limit cycle behaviour and described by a complex Stuart-Landau equation. The shedding may therefore be modelled by such an equation with the addition of a (complex) stochastic forcing term:

$$
\dot{\boldsymbol{x}}=\boldsymbol{\alpha} \boldsymbol{x}-\lambda \boldsymbol{x}|\boldsymbol{x}|^{2}+\boldsymbol{u}(\theta)+\boldsymbol{\sigma} \xi(t),
$$

where all bold symbols denote complex quantities: $\boldsymbol{\alpha}=\alpha_{r}+\mathrm{i} \alpha_{i}$, $\boldsymbol{\lambda}=\lambda_{r}+\mathrm{i} \lambda_{i}, \boldsymbol{u}=u_{r}+\mathrm{i} u_{i}, \boldsymbol{\sigma}=\sigma(1+\mathrm{i})$. Writing $\boldsymbol{x}=a e^{\mathrm{i} \psi},(4)$ becomes,

$$
\begin{aligned}
\dot{a} e^{\mathrm{i} \psi}+\mathrm{i} a \dot{\psi} e^{\mathrm{i} \psi}=\left(\alpha_{r}+\mathrm{i} \alpha_{i}\right) a e^{\mathrm{i} \psi}-\left(\lambda_{r}+\mathrm{i} \lambda_{i}\right) a^{3} e^{\mathrm{i} \psi}+ \\
u_{r}+\mathrm{i} u_{i}+\sigma(1+\mathrm{i}) \xi(t) .
\end{aligned}
$$

Splitting (5) into real and imaginary parts gives equations in each of the magnitude and phase of $\boldsymbol{x}$,

$$
\begin{aligned}
& \dot{a}=\alpha_{r} a-\lambda_{r} a^{3}+ \\
& \quad u_{r} \cos (\psi)+u_{i} \sin (\psi)+\sigma(\cos (\psi)+\sin (\psi)) \xi(t), \\
& \dot{\psi}=\alpha_{i}-\lambda_{i} a^{2}+ \\
& \quad \frac{1}{a}\left(u_{i} \cos (\psi)-u_{r} \sin (\psi)\right)+\frac{\sigma}{a}(\cos (\psi)-\sin (\psi)) \xi(t) .
\end{aligned}
$$

The equation for the amplitude in (6a) is very similar to that for $r$ in (3). In the absence of forcing the dynamics of $a$ would be the same as those for $r$, consisting of bistable switching and fluctuations around a non-zero equilibrium value of $a_{e}= \pm \sqrt{\alpha_{r} / \lambda_{r}}$. For the case again of no input $\boldsymbol{u}$, the equation for the phase $(6 \mathrm{~b})$ describes a process with a mean drift, $\mathrm{E}[\dot{\psi}]=\alpha_{i}-\lambda_{i} \mathrm{E}\left[a^{2}\right]$, and a stochastic forcing. Together these terms impose a mean frequency to the variable $\boldsymbol{x}$, and random fluctuations which give a broadening of the frequency content, as observed in turbulent flows.

A complex Stuart-Landau equation such as (4) is known to be capable of modelling self-excited oscillations ( $\mathrm{Li}$ and $\mathrm{Ju}-$ niper, 2013), and will generate limit-cycle behaviour with a fixed frequency $\omega_{0}=\alpha_{i}-\lambda_{i} \mathrm{E}\left[a^{2}\right]$ under unforced conditions (i.e. $\theta=0$ ). The unique addition here is to include the stochastic term, just as for the bistability modelling, the effect of which is to spread the frequency content around $\omega_{0}$. This generates the broadband oscillations observed for vortex shedding in turbulent flows. A simulated time-series and power spectral density for the imaginary part of $\boldsymbol{x}$ are displayed in figure 2 . The results demonstrate the broadband oscillations that the model generates, demonstrating that the model may qualitatively capture the vortex shedding behaviour observed in many turbulent wake flows.

In addition to the unforced response, of key interest for feedback control is the response of the model in (4) to forcing, 

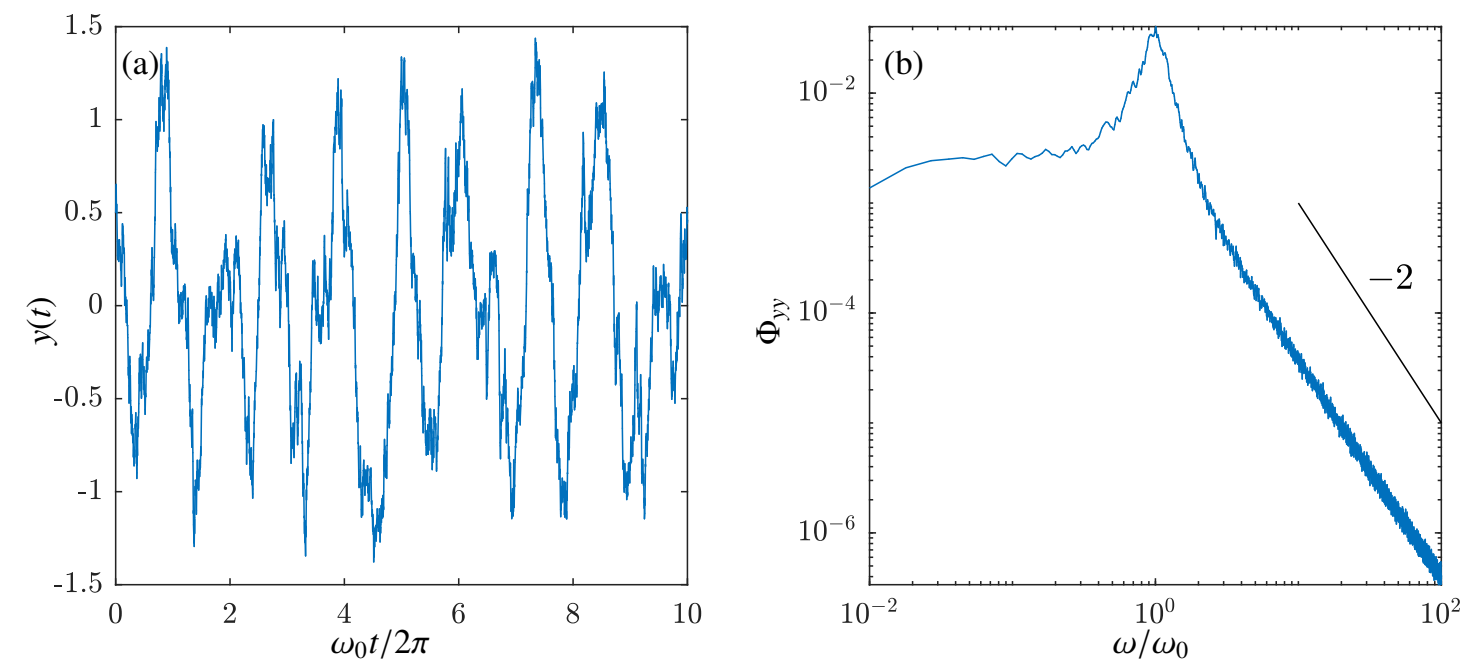

Figure 2: Unforced time series (a) and power spectral density (b) for the variable $y=a \sin (\psi)$ of (4).
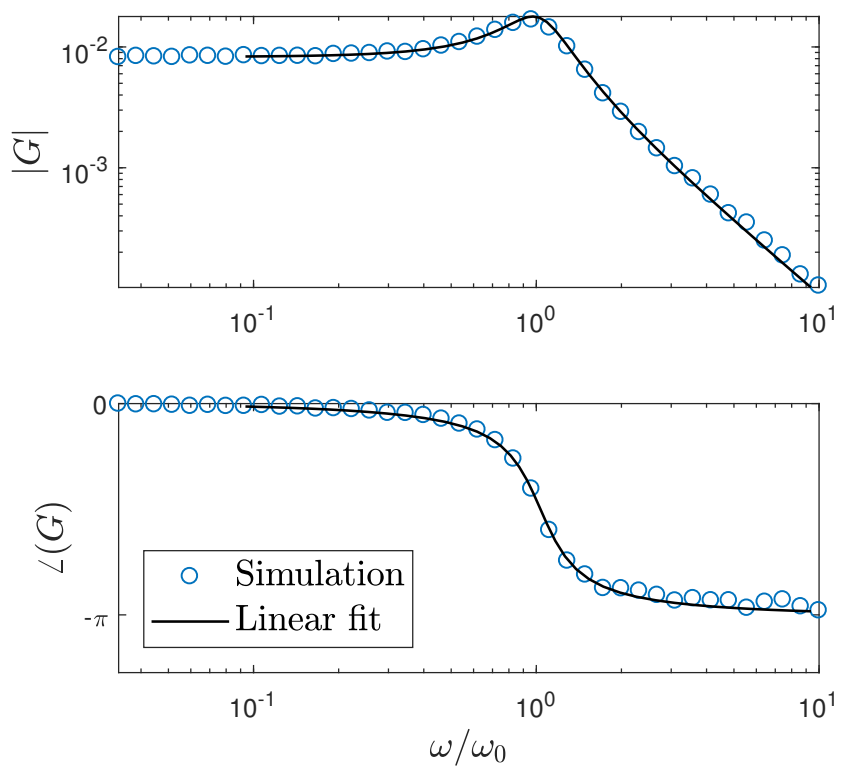

Figure 3: Frequency response of the model (4) between real input $u_{r}=$ $U \sin (\omega t)$ and imaginary output $x_{i}$. Also shown is a linear 2 nd-order fit to the data.

quantified by the frequency response. Applying a purely real $u_{r}(t)=U \sin (\omega t)$, and taking as output the imaginary part of $\boldsymbol{x}$, we may evaluate the frequency response displayed in figure 3. The simulated results here demonstrate that despite the cubic nonlinearity present in the underlying equations, the input-output behaviour may be captured accurately by the frequency response of a linear 2nd-order oscillator.

We may compare the simulated results from (4) with experimental data for the harmonically forced wake. The experimental frequency response for several forcing amplitudes is displayed in figure 4 . The interaction of the flaps with the vortex shedding in the wake is seen for $S t_{H} \approx 0.2$, at which the amplitude of the response is seen to reach a maximum, indicative of
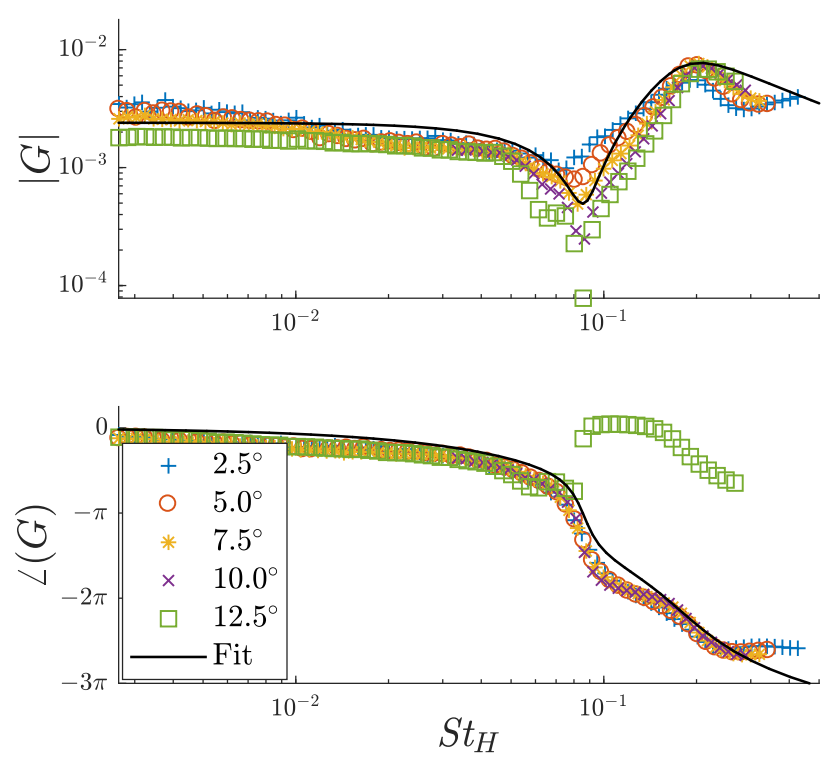

Figure 4: Experimental frequency response between forcing with the top/bottom flaps and the vertical pressure gradient $m_{v}$. For comparison the composite model evaluated as the sum $G_{1}(s)+G_{2}(s)$ is also shown.

a resonance of the vortex shedding. Coinciding with this resonance is a decrease of the phase angle from $-2 \pi$ to around $-3 \pi$. This coincidence of a peak in the amplitude and $-\pi$ shift in the phase is just as observed for the simulated results displayed in figure 3 .

The other obvious comparison to make would be between the unforced spectrum of the model output and that of a typical flow measurement. The measurements required in this work are of base pressure, since this would be the case for a practically implemented system. Unfortunately, it is known that base pressure may correlate poorly with some of the energetic wake features due to the relative spatial locations (Brackston et al., 2014; Brackston, 2017). However a qualitative comparison may be 
made with velocity field data from other studies such as that of Grandemange et al. (2013b); Volpe et al. (2015), which indeed display a broadband peak in the power spectra, just as for our model in figure 2. The spectra of our measured signal will be presented and discussed below in section 4 .

\section{Feedback control}

For the Ahmed body wake, feedback control may proceed along one of two approaches. Either the static asymmetry of the wake may be targeted, aiming to achieve a more symmetric wake on average, or the unsteadiness associated with vortex shedding may be targeted. The former of these strategies was applied in the lateral dimension in which the bistability is present, and was found to be effective at providing efficient drag reduction (Brackston et al., 2016). The latter strategy is that applied here in the vertical dimension. We will begin this work by giving our interpretation of the models and experimental data from a control systems perspective, before describing the control design methodology. The results of the feedback control will then be analysed in detail.

\subsection{Frequency Response Interpretation}

The models described above give nonlinear stochastic equations for the dynamics of the bistability caused by the SB mode and the quasi-oscillatory vortex shedding. Despite the nonlinearity of the underlying models and flow, it is important to note that we can still deduce linear input-output behaviour in the form of the frequency response. We may therefore hope to explain many of the features of the experimental frequency response of figure 4, based upon the models for the flow.

One significant feature of the experimental frequency response displayed in figure 4 is the sharp trough in $|G|$ and the accompanying phase shift seen at $S t_{H} \approx 0.09$. This behaviour is indicative of what are known as right-half-plane zeros in control theory, and can result from a cancellation between two independent sets of dynamics, dominant over different frequency ranges (see e.g. Skogestad and Postlethwaite, 2005, 5.7). Such a pair of dynamics may indeed result from the combined ability of forcing flaps to alter both the orientation of the separation bubble at low frequencies, and interact with the oscillatory vortex shedding at higher frequencies. As discussed above, we may expect the frequency response of the vortex shedding to act like a 2nd-order linear system. For the low frequency quasi-static deflection of the wake we may expect the response to be large at very low frequencies, corresponding to the ability of the flaps to deflect the mean flow. At higher frequencies this behaviour would decay due to a limited reaction speed of the wake, and may therefore be modelled by a simple 1st-order low-pass system. We therefore have the following transfer functions:

$$
\begin{gathered}
G_{1}(s)=g_{1} \frac{1}{1+s T_{1}}=\frac{n_{1}}{d_{1}}, \\
G_{2}(s)=g_{2} \frac{1}{1+2 \zeta s T_{2}+s^{2} T_{2}^{2}}=\frac{n_{2}}{d_{2}} .
\end{gathered}
$$

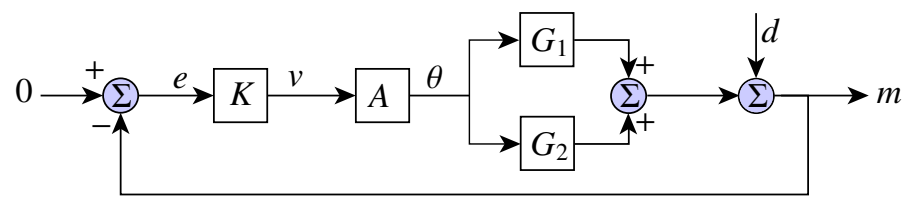

Figure 5: A schematic of the feedback control loop comprising the wake $G_{1,2}$, actuator $A$ and feedback controller $K . G_{1}$ captures the low-frequency dynamics of the bistability (lateral) or near-wake deflection (vertical), while $G_{2}$ captures the vortex shedding and shear layer dynamics at higher frequencies.

In order for a cancellation to occur, the two transfer functions must be added together, $G=G_{1}+G_{2}$. This is based on the idea that the two features are essentially independent, therefore the total response of the wake may be considered as the sum of the two responses. The poles of the resulting transfer function $G$ will be the poles of the two constituent transfer functions, however the zeros will not be the same but are instead given by the roots of

$$
\begin{aligned}
n(s) & =g_{1}\left(1+2 \zeta s T_{2}+s^{2} T_{2}^{2}\right)+g_{2}\left(1+s T_{1}\right) \\
& =g_{1}+g_{2}+\left(2 g_{1} \zeta T_{2}+g_{2} T_{1}\right) s+g_{1} T_{2}^{2} s^{2} \\
& =g\left(1+2 \zeta_{z} T_{z} s+T_{z}^{2} s^{2}\right) .
\end{aligned}
$$

The criteria for the pair of RHP zeros observed experimentally are therefore,

$$
\begin{array}{r}
g_{1}+g_{2}=g>0, \\
2 g_{1} \zeta T_{2}+g_{2} T_{1}=2 g \zeta_{z} T_{z}<0, \\
g_{1} T_{2}^{2}=g T_{z}^{2}>0 .
\end{array}
$$

These criteria may be satisfied by appropriate choice of the parameters, and furthermore the properties of the response may be chosen to fit with observations. For example, if we specify the DC gain $g$, the properties of the zeros (specified by $T_{z}$ and $\zeta_{z}$ ) and the properties of the poles (specified by $\zeta$ and $T_{2}$ ), we have enough information to determine all remaining parameters. The black line displayed in figure 4 is just such a fit composed of the sum of 1 st and 2 nd-order linear systems. This fit can be seen to capture the key behaviour in both magnitude and phase.

A schematic of the block diagram for the system is displayed in figure 5. In this schematic the wake is modelled as the sum of two transfer functions: $G_{1}$ capturing the response of the lowfrequency behaviour of the static wake deflection and $G_{2}$ capturing the interaction of the flaps with the vortex shedding. The disturbances $d$ describe all other unmodelled dynamics entering the measurement $m$. The transfer function $A$ describes the dynamics of the actuators while $K$ is the feedback controller. This schematic of the system and the interpretation underpinning it may subsequently be used in the design of feedback controllers.

\subsection{Implications for feedback control}

Having found that the observed frequency response may be explained by the models of section 3 and that the behaviour is indicative of RHP zeros, it is important to consider the implications of these observations for feedback control design. Intuitively, it is clear that for the range of frequencies in which the 
measured response is very small, it will be difficult to provide effective control. Further to this, the large decrease in phase angle associated with the presence of RHP zeros may be considered similar to a delay in the response. It may also be intuitive that if the response of the system is consistently delayed relative to the input, it will be difficult to provide the correct input in response to unpredictable disturbances. For a more rigorous understanding of the effect of RHP zeros we can look at what is known as the sensitivity function $S(\mathrm{i} \omega)$.

The sensitivity function defines the expected ratio of measured fluctuations, with and without control. Considering the system displayed in the block diagram of figure 5 , we can first define a "loop" transfer function as $L=\left(G_{1}+G_{2}\right) A K$. If no control is present then the measurement $m$ will consist purely of the disturbances $d$. With the negative feedback loop in place it may be shown that,

$$
\begin{aligned}
m & =\frac{1}{1+L} d, \\
& =S d,
\end{aligned}
$$

where we define the sensitivity function as $S=1 /(1+L)$. This transfer function describes, as a function of frequency, how the exogenous disturbances $d$ are amplified or attenuated by the action of the feedback control. For a system with no RHP poles but a complex conjugate pair of RHP zeros at $z=x \pm$ iy it may be shown that the following integral must be satisfied (Freudenberg and Looze, 1985)

$$
\int_{0}^{\infty} \ln |S(\mathrm{i} \omega)| w(z, \omega) \mathrm{d} \omega=0,
$$

where $^{1}$

$$
w(z, \omega)=\frac{x}{x^{2}+(y-\omega)^{2}}+\frac{x}{x^{2}+(y+\omega)^{2}} .
$$

This is similar to the classical waterbed formula from Bode (1945), the difference being the frequency dependent weighting function $w(z, \omega)$. The weighting function is large near to the frequency of the zeros and decays away from this frequency. The result of this is that any attenuation $(S<1)$ in a frequency range near to that of the zeros must be compensated by an amplification $(S>1)$ within a similar range. As we shall see below, such amplification is often detrimental with regard to drag reduction, therefore the limitation of (12) is of key importance and will impose limitations to the control efficacy.

\subsection{Control design}

The control strategy applied here seeks to target fluctuations directly, specifically aiming to reduce the fluctuations at $S t \approx 0.2$ which are associated with the vortex shedding. To do this we may design controllers based on the sensitivity function $S(\omega)$. This is an approach that has been successfully applied before in fluid flows (Dahan et al., 2012; Dalla Longa et al., 2017;

\footnotetext{
${ }^{1}$ Note that the actual values of this function are unimportant, what matters is the variation with frequency.
}
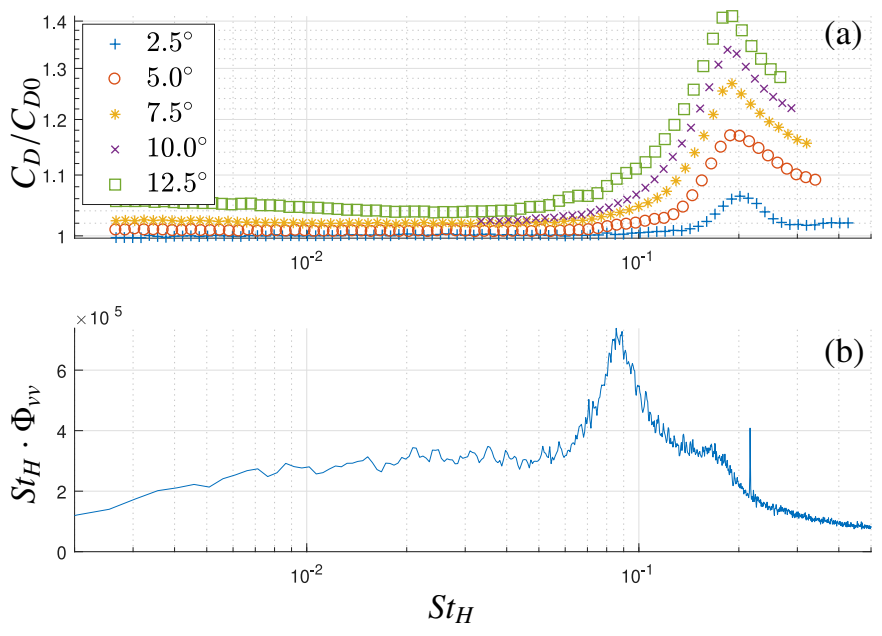

Figure 6: Drag change with open-loop forcing frequency (a) and PSD of the vertical pressure metric $m_{v}$ for the unforced flow (b).

Evstafyeva et al., 2017), and may be based purely upon empirical linear models of the input-output behaviour. In this case we therefore do not seek to obtain a linear model by linearising (4) directly, but rather make use of the frequency response characteristics discussed above and displayed in figure 4. However, it is important to note that the form of the frequency response that we fit is consistent with the properties of the model, as discussed above. For the application of this approach using the top/bottom flaps $G_{1}$ is a simple 1st-order low-pass filter while $G_{2}$ is a second order oscillator, chosen to fit the frequency response in figure 4 . The pressure measurement $m$ is taken as the vertical pressure metric, as defined in section 2 .

A starting point for the design of fluctuation-suppressing controllers is to examine the unforced spectrum and the drag change that open-loop forcing generates, in order to identify the frequency range of interest. Figure 6 displays displays the drag change observed under the influence of open-loop harmonic forcing as well as the PSD of the pressure metric $m_{v}$ for the baseline case in which top/bottom flaps are held fixed. The flaps are seen to interact very strongly with the vortex shedding for $S t_{H} \approx 0.2$. This frequency range is seen to approximately coincide with a "hump" in the premultiplied spectra of figure 6(b). While this hump is notably unpronounced, such an observation is not unusual for the base pressure signal in high Reynolds number three-dimensional flows (Rigas et al., 2014). Furthermore, the frequency of the shedding is also consistent with previous results for the Ahmed body from Grandemange et al. (2013b) and Volpe et al. (2015).

Motivated by the spectra and observed drag changes displayed in figure 6 we may design controllers aiming to provide suppression in a frequency range around $S t_{H} \approx 0.2$. Given the relative complexity of the system we are trying to control, it is desirable to use one of the many automated control-design algorithms available. We choose to use what is known as $\mathcal{H}_{\infty}$ synthesis, using the MatLAB function hinfsyn. As explained below, this allows automated shaping of the sensitivity function $S$, while attempting to maintain predicted stability of the closed-loop system. 


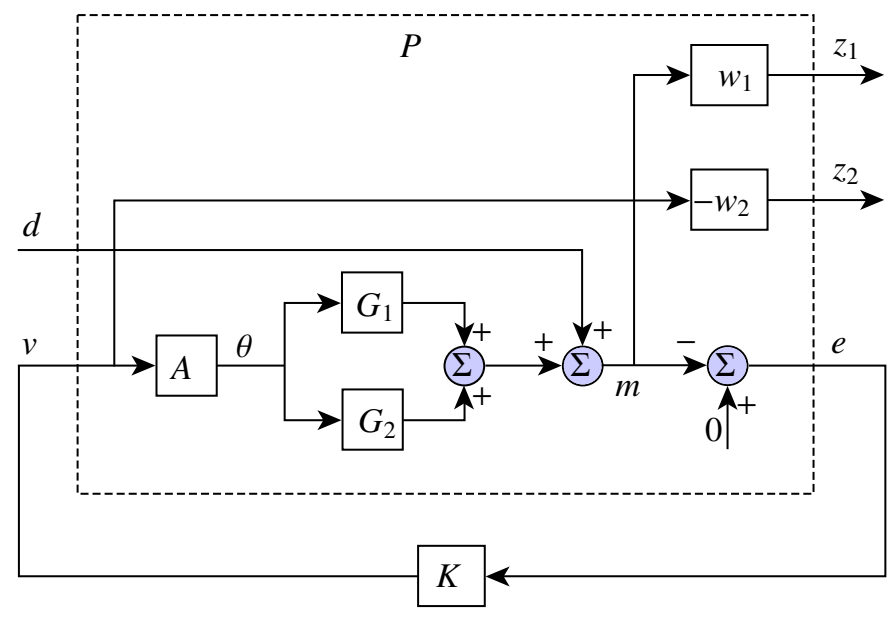

Figure 7: A schematic of the feedback control loop of figure 5, reformatted for the $\mathcal{H}_{\infty}$ synthesis problem. The additional blocks $w_{1}$ and $w_{2}$ are weighting functions that are chosen in order to specify the desired properties of the closedloop system.

Application of $\mathcal{H}_{\infty}$ synthesis requires us to rewrite our model for the system, in order to conform with the general $\mathcal{H}_{\infty}$ synthesis problem. Figure 7 displays a schematic of the feedback control set-up, reformulated appropriately. The system is shown as a generalised plant $P$ and controller $K$ connected in mutual feedback. The generalised plant $P$ has two inputs: the voltage $v$ applied to the motors and the exogenous disturbance $d$. We also choose for $P$ to have three outputs: the error signal $e$ and two additional errors $z_{1}$ and $z_{2}$. These additional error signals are chosen such that for the closed-loop system they are related to the inputs via the following transfer functions:

$$
\begin{aligned}
& z_{1}=w_{1}\left(\frac{1}{1+G A K}\right) d=w_{1} S d, \\
& z_{2}=w_{2}\left(\frac{K}{1+G A K}\right) d=w_{2} K S d .
\end{aligned}
$$

The $\mathcal{H}_{\infty}$ synthesis algorithm then finds a $K$ that minimises the $\cos t \gamma$, such that for all frequencies $\omega$,

$$
\begin{aligned}
w_{1}(\mathrm{i} \omega) S(\mathrm{i} \omega) & \leq \gamma, \\
w_{2}(\mathrm{i} \omega) K(\mathrm{i} \omega) S(\mathrm{i} \omega) & \leq \gamma
\end{aligned}
$$

The power of the $\mathcal{H}_{\infty}$ control-design process described above is that by choosing the frequency response of the weighting functions $w_{1}$ and $w_{2}$ we can shape directly the closed-loop properties of the system; something that is otherwise challenging to do in an ad-hoc manner. More specifically, we choose $w_{1}$ to be large over the frequency range in which we require fluctuation suppression. If we perform the design process specifying only $w_{1}$, overly aggressive control action may result, characterised by a controller with very high gain at some frequency. We can therefore employ the weighting function $w_{2}$ to prevent such behaviour by first finding the frequency at which $K$ is large, then choosing $w_{2}$ to be large at this same frequency. Our controldesign procedure is therefore a two-stage process where we first specify only $w_{1}$, then specify both $w_{1}$ and $w_{2}$.

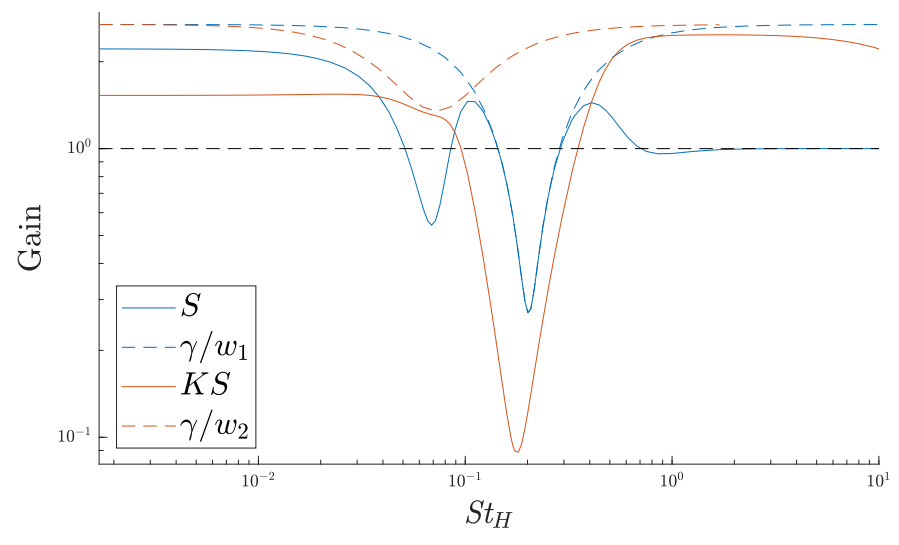

Figure 8: Illustration of a particular control design showing the weighting functions $w_{1}, w_{2}$ and the corresponding closed-loop transfer functions $S, K S$.

To further clarify how the control-design process works, figure 8 displays the transfer functions from a particular controller design. Displayed are the scaled weighting functions $\gamma / w_{1}$ and $\gamma / w_{2}$ and the corresponding closed-loop transfer functions $S$ and $K S$. As displayed, $1 / w_{1}$ is chosen to be a "notch" function, dipping in a particular frequency range of $S t_{H} \approx 0.2$. The $\mathcal{H}_{\infty}$ control-design generates a $K$ such that $S$ is also small in this same frequency range, but is necessarily large elsewhere. The particular frequency and the width of the notch specified by $1 / w_{1}$ can be varied and a large number of variants were designed. The two-stage process of control design is detailed further in algorithm 1 .

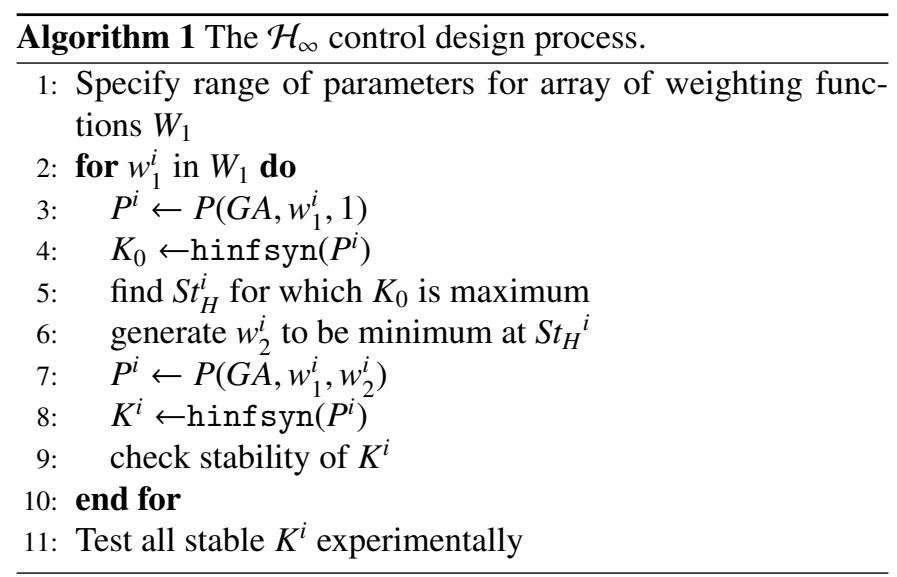

Using algorithm 1, we design 123 different controllers to test experimentally. Each controller has an expected sensitivity function designed to have a trough that leads to fluctuation suppression over a particular frequency range. All controllers are therefore similar in form to that shown in figure 8 , the difference between the controllers being the frequency and sharpness of the trough.

\subsection{Control results and analysis}

Following the application of the procedure outlined above, a total of $N=123$ controllers were tested, all achieving attenuation and amplification of fluctuations over different frequency 


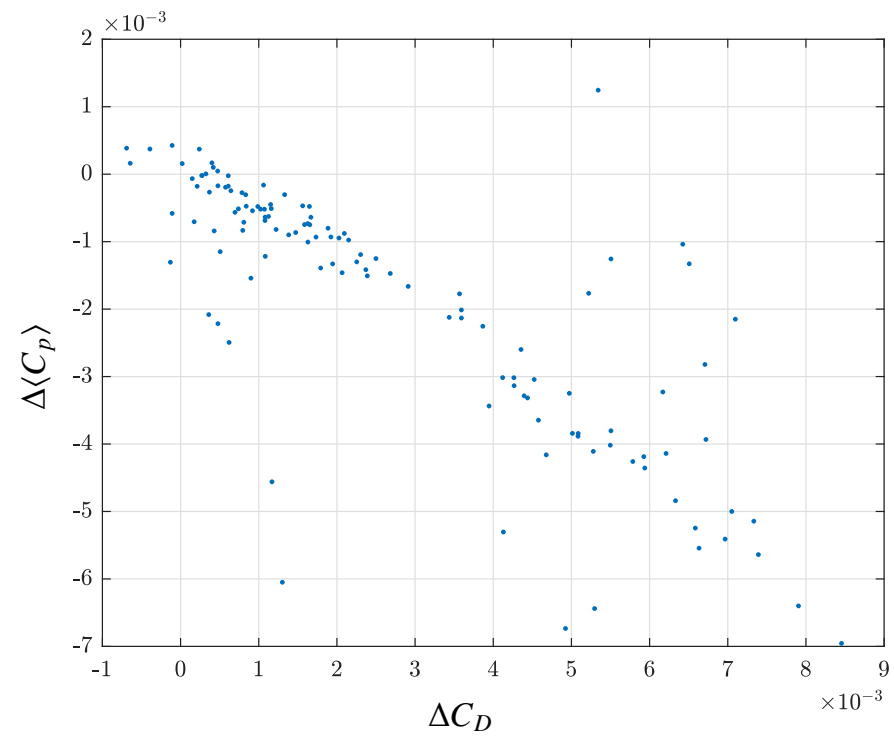

Figure 9: Scatter plot of the drag and base pressure changes achieved by all of the tested controllers. Positive $\Delta C_{D}$ corresponds to a drag increase.

ranges. The performance of these controllers, quantified by the achieved changes in the drag coefficient $C_{D}$ and spatially averaged base pressure coefficient $\left\langle C_{p}\right\rangle$, is displayed in figure 9. Only five of the controllers give a measured drag reduction, and for all of these the drag change is within the experimental uncertainty. The applied strategy is therefore unsuccessful in achieving a drag reduction.

To exemplify the effect that a controller can have on the flow, the PSD for the pressure metric $m_{v}$ under the action of a particular controller is displayed in figure 10. It is clear that for this particular controller, suppression of the measured fluctuations is achieved around the shedding frequency of $S t_{H} \approx 0.2$. Also clear is the large amplification that is imposed at other frequencies, just as was also found for bistability control (Brackston et al., 2016). From the changes to $C_{D}$ and $\left\langle C_{p}\right\rangle$, we know that the net effect of these changes is to increase the drag, although from only a single example it is not possible to decouple the beneficial and detrimental effects of the control. It is likely that the additional fluctuations lead to the drag increase. Unfortunately, some amplification is essential due to the waterbed effect and furthermore, is restricted to a particular frequency range by the presence of the right-half-plane zeros in the frequency response (see for example Skogestad and Postlethwaite, 2005). Given that none of the controllers proved successful at drag reduction, it is difficult to determine whether the fluctuation suppression is not beneficial, or if a positive effect is simply offset by the impact of amplification at other frequencies.

Given the control results we wish to know if there is something inherently wrong with the strategy of measurement attenuation and amplification, or if drag reduction would be possible given the correct choice of controller. While for any particular controller it is only possible to observe the net impact of the changes in measured fluctuations, leveraging the information from many controllers we may hope to learn the average effect of attenuation or amplification as a function of frequency.

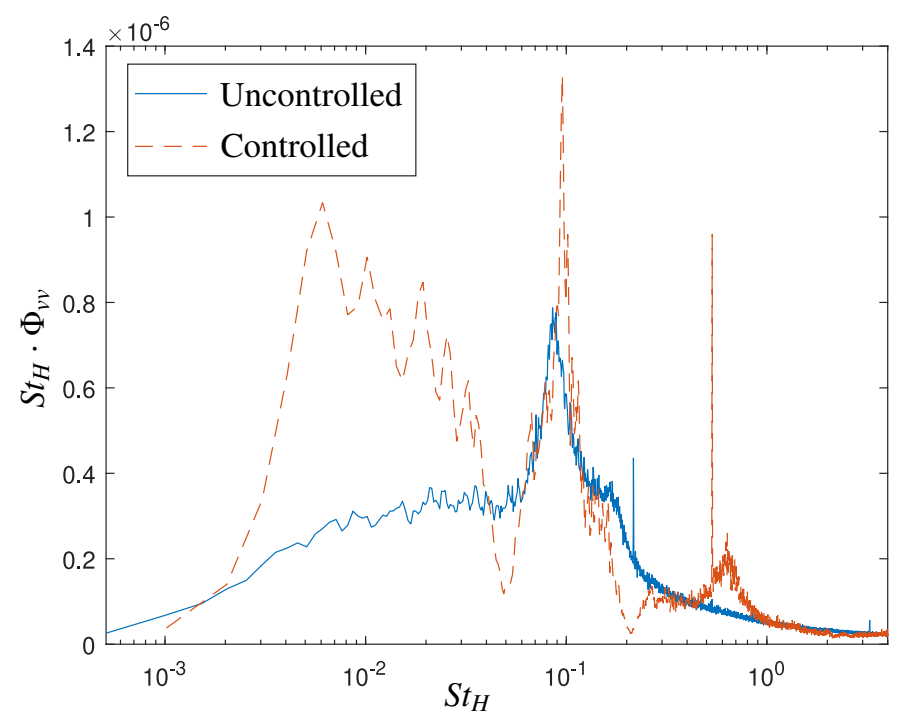

Figure 10: PSD of the vertical pressure gradient $m_{v}$ for a particular controller. Suppression is achieved around $S t_{H} \approx 0.2$, however the drag is not reduced.

From the experimental implementations we have $N=123$ controllers, each with a predicted sensitivity function $S_{i}$ and drag change $D_{i}$. Since the predictions of the $S_{i}$ will not be exactly borne out in the results, we define the experimentally determined spectral changes as,

$$
M_{i}(\omega)=\ln \left(\frac{\widetilde{m}_{v}^{i}(\omega)}{\widetilde{m}_{v}^{0}(\omega)}\right)
$$

Here $\widetilde{m}_{v}^{i}$ and $\widetilde{m}_{v}^{0}$ are the magnitude of the Fourier transform of $m_{v}$ for the $i$ 'th controller and baseline case respectively. Due to the use of the logarithm in (18), the parameter $M_{i}$ has the property that for the case of no control, it will be expected to have a value of zero for all frequencies.

As a first try, we may look to fit a linear relationship between $M_{i}(\omega)$ and $D_{i}$. Such a relationship can be defined by the linear integral relation,

$$
D(M)=\int_{0}^{\infty} F(\omega) M(\omega) \mathrm{d} \omega,
$$

where $F(\omega)$ is the frequency dependent mapping function. Such a function is intended to apply for all the controllers tested here, but would likely be specific to the particular experimental geometry and the measurements through which $M(\omega)$ is evaluated.

We may hypothesize that $F$ be large and positive around $S t_{H} \approx 0.2$, as this would be consistent with our expectation that amplification/attenuation of the vortex shedding would lead to drag increases/decreases respectively. At this point it is worth noting the similarity between (19) and the waterbed constraint of (12). For the case that the sensitivity function is entirely accurate, $M(\omega)=\ln |S(\omega)|$, so we may therefore gain some insight from the differences between $F(\omega)$ and the waterbed weighting function $w(z, \omega)$, as defined in (13). For example, if $F(\omega)=w(z, \omega)$ and the relation of (19) were true, it would 
be impossible to cause drag changes through the action of feedback control. An alternative for a "frequency independent" flow might be a constant $F(\omega)=c$. In this case drag reduction could be achieved by placing amplification in the region where $w(z, \omega)$ is large, thereby achieving large suppression over other frequencies. In practice neither of these seem likely to be the case, but as examples give some of the possible implications arising from the particular form of the function $F(\omega)$.

The linear fitting may be estimated via a least-squares minimisation, seeking to approximate $F(\omega)$ in a piecewise manner. This procedure consists of finding a vector $\hat{F}$ that minimises the error between the observed drag and that predicted by (19). The frequency bins of this discretized weighting function are chosen to be logarithmically spaced, as this gives the fairest way to capture possible changes at all frequencies within the range of interest. The weighting function is displayed in figure 11(a), plotted alongside the open-loop spectrum for reference. Figure 11(b) shows the relationship between the true drag change induced by each controller, and that given by our approximation (19). These results demonstrate a good agreement, suggesting that we may have some faith in the best-fit vector $\hat{F}$ and the relationship described by (19).

At very low frequencies, $\hat{F}$ is seen to vary between large positive and negative values. This is likely a result of over-fitting and the limited frequency resolution at this end of the spectrum, so may not necessarily have any physical significance. A possibly more significant positive value is seen for $S t_{H} \approx 0.09$. Conversely for $S t_{H} \approx 0.2$, the value of $\hat{F}$ is fairly small, although still positive. This would imply that, in fact, attenuation/amplification around the frequency of the shedding has relatively little effect, compared with at frequencies approximately half that of the shedding. Regardless of this, the form of the weighting function may give some clue as to why the control was generally unsuccessful, and is displayed in figure 12 . The peak close to $S t_{H} \approx 0.09$ coincides approximately with that of $w(z, \omega)$ which is in turn the location of the RHP zeros in the frequency response. This means that it is practically very difficult to design a stable controller that provides suppression around this frequency. Moreover, most of the controllers tested in this study aimed at providing suppression at frequencies higher than $S t_{H}=0.1$ so generally generated amplification over this frequency range, therefore if the form of $\hat{F}$ is to be trusted, it is not surprising that these controllers failed to achieve drag reduction. Further work targeting lower frequency ranges may therefore prove more successful.

It is finally important to note the limitations to the above analysis approach. The founding assumption of a linear relation between $D$ and $M$ is of course unlikely to hold in practice, although is a natural first approximation. This is especially the case given that we are considering both amplification and attenuation under the same relationship. The main issue is that since none of the controllers achieved any real drag reduction, drawing insight towards more effective controllers effectively involves extrapolation of the observed trends into unknown operating conditions. The linear basis for the relationship may then be insufficient. It is also possible that the range of controllers tested had insufficiently varying characteristics for the full frequency-dependent form of $\hat{F}$ to be determined. This problem makes the preceding arguments for the poor performance somewhat circular: all controllers increased the drag and many gave amplification near $S t_{H} \approx 0.1$, possibly therefore leading to the form of $\hat{F}$, rather than the drag increases being the result of it. More thorough analysis ought really to include an estimate of the uncertainty in $\hat{F}$, based upon the range of spectral changes observed within each frequency bin. Despite these limitations, it seems likely that if due care is taken to explore more fully the frequency range of interest and to evaluate the statistical uncertainty, the above suggested method should provide a more rigorous basis on which to design controllers via the sensitivity approach.

\section{Concluding remarks}

We have presented a stochastic modelling approach suitable for some of the coherent structures observed in threedimensional bluff body wakes, here focussing on the vortex shedding. This approach takes the equation describing the underlying bifurcation observed at low $R e$, and adds a stochastic term to model phenomenologically the effect of turbulent fluctuations on the large-scale coherent structures. For the case of the SB mode, this approach has already been shown to be effective at modelling the resultant bistable wake behaviour. We have additionally demonstrated here that the vortex shedding in turbulent wakes may be qualitatively modelled by a complex Stuart-Landau equation with stochastic forcing, and furthermore that such a model captures the typical forced response of the wake.

In order to design feedback controllers for the flow it is important to have models that accurately capture the complete forced response. We find that by expressing the response of the wake as the summation of a first and second order linear system, the frequency response may be accurately captured. Moreover, such a frequency response is consistent with the stochastic models for the vortex shedding. One of the key experimentally observed phenomena is a sharp trough in the frequency response indicative of what are known as right-half-plane zeros, the presence of which imposes limitations on our ability to provide tight control over a wide frequency range.

Given a fitted frequency-domain model of the flow, we presented a feedback-control approach that targets the measured fluctuations in the wake directly. This control approach takes advantage of the linear frequency response implied by the nonlinear stochastic models, designing controllers using a quasiautomated shaping of the sensitivity function. Measured fluctuations of the vertical pressure gradient may be suppressed over the frequency range of the vortex shedding, although no controllers were found that could give a resultant drag reduction. The success may be limited by the amplification of measured fluctuations over other frequency ranges, a problem compounded by the presence of the RHP zeros in the frequency response. As discussed, the zeros may be a result of the combined ability of the flaps to influence both low-frequency quasistatic features, and the oscillatory vortex shedding. This may therefore represent a limitation of forcing flaps as an actuator 

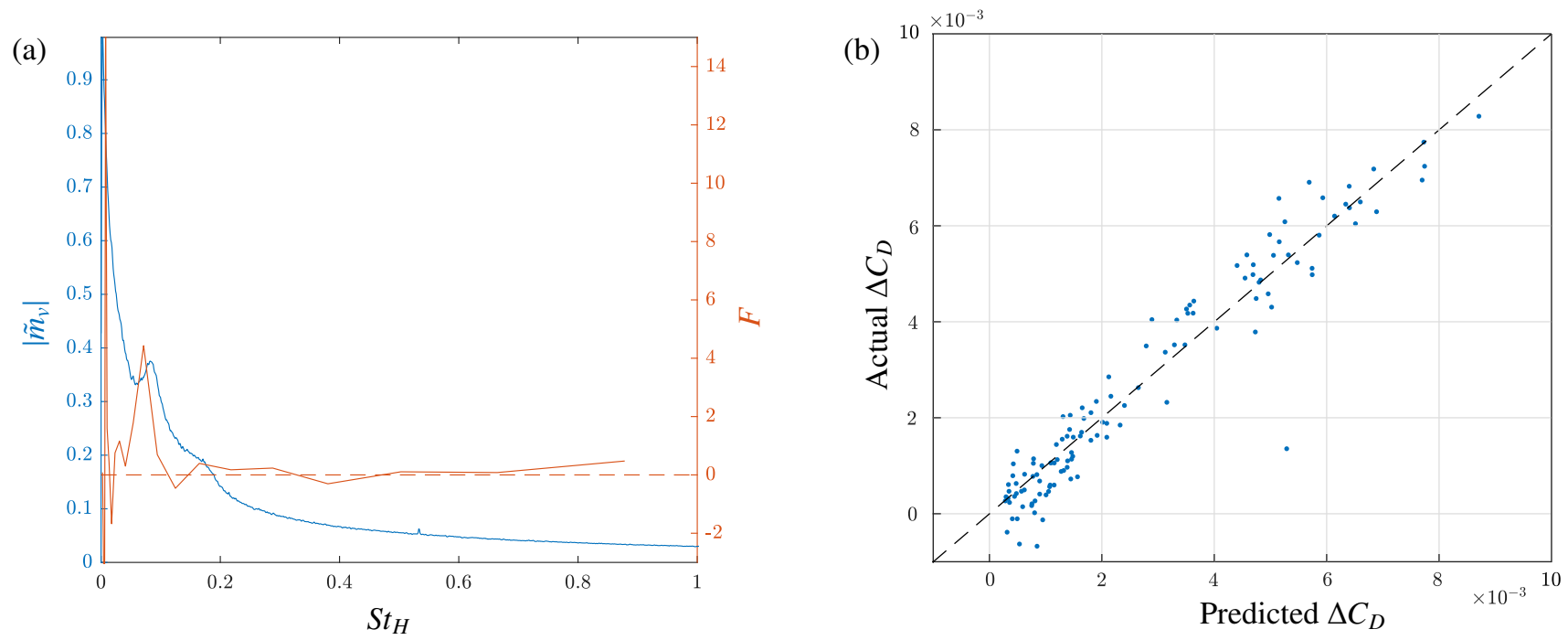

Figure 11: (a) A comparison of the best-fit weighting function $\hat{F}$ with the uncontrolled spectrum, and (b) a comparison of the actual and predicted drag changes from the least-squares fit.

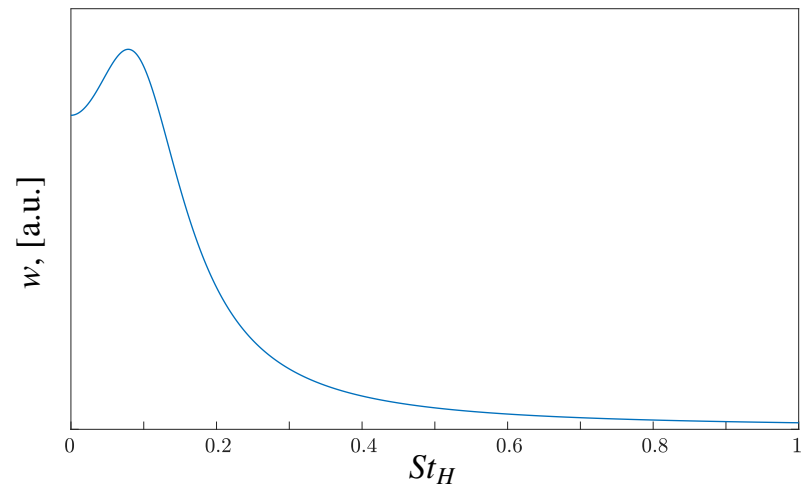

Figure 12: The frequency weighting function $w(z, \omega)$, displayed as a function of $S t_{H}$.

for this flow. The related limitation imposed by the waterbed constraint of (12), is one that applies in the context of the linear feedback-control methods employed in this work. It is perceivable that through the use of more complex nonlinear methods such as sum-of-squares optimisation (Chernyshenko et al., 2014; Lasagna et al., 2016), this limitation could to some extent be circumvented. Such approaches would require an accurate (nonlinear) model for the system, of which the models of section 3 may be a suitable example.

An improved understanding of the fundamental limitations to the control of turbulent flows is of crucial importance in the targeted development of new methods for drag reduction. Despite the ultimately unsuccessful control strategy presented here, we hope that the detailed control design and analysis methodologies may prove useful in this endeavour.

\section{Achnowledgements}

This work was supported by EPSRC grant no 1370746 . We are also grateful to Dr Juan Marcos García de la Cruz Lopez and Dr Georgios Rigas for helpful discussions.

\section{References}

Ahmed, S., Ramm, G., Faltin, G., 1984. Some salient features of the timeaveraged ground vehicle wake. SAE Tech. Rep. 840300, Society of Automotive Engineers.

Barros, D., Borée, J., Noack, B. R., Spohn, A., 2016. Resonances in the forced turbulent wake past a 3D blunt body. Phys. Fluids 28, 065104.

Bode, H. W., 1945. Network Analysis and Feedback Amplifier Design. Van Nostrand, New York.

Brackston, R. D., August 2017. Feedback control of three-dimensional bluff body wakes for efficient drag reduction. $\mathrm{PhD}$, Imperial College London. URL http://hdl . handle.net/10044/1/52406

Brackston, R. D., García de la Cruz, J. M., Wynn, A., Rigas, G., Morrison, J. F., 2016. Stochastic modelling and feedback control of bistability in a turbulent bluff body wake. J. Fluid Mech. 802, 726-749.

Brackston, R. D., Wynn, A., Morrison, J. F., 2014. Observability of the turbulent wake behind an axisymmetric bluff body. In: Bulletin of the American Physical Society, 67th Meeting of the DFD.

Cadot, O., Evrard, A., Pastur, L., 2015. Imperfect supercritical bifurcation in a three-dimensional turbulent wake. Phys. Rev. E 91, 063005.

Chernyshenko, S. I., Goulart, P., Huang, D., Papachristodoulou, A., 2014. Polynomial sum of squares in fluid dynamics: a review with a look ahead. Phil. Trans. R. Soc. A 372.

Dahan, J. A., Morgans, A. S., Lardeau, S., 2012. Feedback control for formdrag reduction on a bluff body with a blunt trailing edge. J. Fluid Mech. 704, 360-387.

Dalla Longa, L., Morgans, A. S., Dahan, J. A., 2017. Reducing the pressure drag of a D-shaped bluff body using linear feedback control. Theor. Computat. Fluid Dyn., 1-11.

Evrard, A., Cadot, O., Herbert, V., Ricot, D., Vigneron, R., Délery, J., 2015. Fluid force and symmetry breaking modes of a 3D bluff body with a base cavity. J. Fluid Struct. 61, 99-114.

Evstafyeva, O., Morgans, A. S., Dalla Longa, L., 2017. Simulation and feedback control of the Ahmed body flow exhibiting symmetry breaking behaviour. J. Fluid Mech. 817, R2.

Ffowcs Williams, J. E., Zhao, B. C., 1989. The active control of vortex shedding. J. Fluid Struct. 3 (2), 115-122.

Flinois, T. L. B., Morgans, A. S., 2016. Feedback control of unstable flows: a direct modelling approach using the eigensystem realisation algorithm. J. Fluid Mech. 793, 41-78. 
Freudenberg, J. S., Looze, D. P., 1985. Right Half Plane Poles and Zeros and Design Tradeoffs in Feedback Systems. IEEE Transactions on Automatic Control AC-30 (6), 555-565.

García de la Cruz, J. M., Oxlade, A. R., Morrison, J. F., 2017. Passive control of base pressure on an axisymmetric blunt body using a perimetric slit. Phys. Rev. Fluids 2, 043905

Grandemange, M., Cadot, O., Gohlke, M., 2012. Reflectional symmetry breaking of the separated flow over three-dimensional bluff bodies. Phys. Rev. E 86,035302 .

Grandemange, M., Gohlke, M., Cadot, O., 2013a. Bi-stability in the turbulent wake past parallelepiped bodies with various aspect ratios and wall effects. Phys. Fluids 25, 095103.

Grandemange, M., Gohlke, M., Cadot, O., 2013b. Turbulent wake past a threedimensional blunt body. Part 1. Global modes and bi-stability. J. Fluid Mech. 722, 51-84.

Illingworth, S. J., 2016. Model-based control of vortex shedding at low reynolds numbers. Theor. Comput. Fluid Dyn. 30 (5), 429-448.

Illingworth, S. J., Naito, H., Fukagata, K., 2014. Active control of vortex shedding: An explanation of the gain window. Phys. Rev. E 90, 043014.

Jackson, C. P., 1987. A finite-element study of the onset of vortex shedding in flow past variously shaped bodies. J. Fluid Mech. 182, 23-45.

Lasagna, D., Huang, D., Tutty, O. R., Chernyshenko, S., 2016. Sum-of-squares approach to feedback control of laminar wake flows. J. Fluid Mech. 809, 628-663.

Li, L. K. B., Juniper, M. P., 2013. Lock-in and quasiperiodicity in a forced hydrodynamically self-excited jet. J. Fluid Mech. 726, 624-655.

Li, R., Barros, D., Borée, J., Cadot, O., Noack, B. R., Cordier, L., 2016. Feedback control of bimodal wake dynamics. Exp. Fluids 57, 158

Pastoor, M., Henning, L., Noack, B. R., King, R., Tadmor, G., 2008. Feedback shear layer control for bluff body drag reduction. J. Fluid Mech. 608, 161196.

Rigas, G., Morgans, A. S., Brackston, R. D., Morrison, J. F., 2015. Diffusive dynamics and stochastic models of turbulent axisymmetric wakes. J. Fluid Mech. 778, R2.

Rigas, G., Morgans, A. S., Morrison, J. F., 2017. Weakly nonlinear modelling of a forced turbulent axisymmetric wake. J. Fluid Mech. 814, 570-591.

Rigas, G., Oxlade, A. R., Morgans, A. S., Morrison, J. F., 2014. Lowdimensional dynamics of a turbulent axisymmetric wake. J. Fluid Mech. 755, R5.

Roussopoulos, K., Monkewitz, P. A., 1996. Nonlinear modelling of vortex shedding control in cylinder wakes. Physica D: Nonlinear Phenomena 97 (1), 264-273.

Skogestad, S., Postlethwaite, I., 2005. Multivariable Feedback Control. Analysis and Design, 2nd Edition. John Wiley \& Sons Inc.

Son, D., Jeon, S., Choi, H., 2011. A proportional-integral-differential control of flow over a circular cylinder. Philos. Trans. A. Math. Phys. Eng. Sci. 369 (1940), 1540-55.

Volpe, R., Devinant, P., Kourta, A., 2015. Experimental characterization of the unsteady natural wake of the full-scale square back Ahmed body: flow bistability and spectral analysis. Exp. Fluids 56, 99. 